\title{
HIGH SPEED AND HIGH ACCURACY POSITIONING OF PNEUMATIC CYLINDER WITH IMPROVED PULSE DRIVING METHOD
}

\author{
Nobutaka KIKU, Katsuya INUZUKA \\ and Kazuya NAKASHIMA
}

3rd development department.

Aisin Seiki Co., Ltd.

2-1 Asahi-machi, Kariya, Aichi, 448 Japan

\begin{abstract}
The position control of a pneumatic cylinder at high speed and high accuracy is very difficult due to nonlinearities of its movement because of the air compressibility or the sliding frictions. In this study, above problems, the improved pulse driving control method is proposed to overcome. The position is controlled by the pulse driving high response on-off valves. In this control method, the phase plane trajectory is confined on the switching line using differential pressure. This position control is executed, the dynamic response and the position accuracy are experimentally examined.

As a result, obtained characteristics are so satisfactory that the efficiency of the proposed control method are ascertained.

And we have developed a positioning controller of a pneumatic cylinder with this control method. It has been used as a pneumatic servo robot in an actual product assembly lines.
\end{abstract}

\section{KEY WORDS}

Pneumatic Cylinder, Pneumatic Servo Robot, Pulse Driving Method, Position Control

\section{INTRODUCTION}

In recent years, rodless cylinders have been used widely as pneumatic actuators for conveyance work in industrial fields.

Rodless cylinders have features including its spacesaving and long-stroke performance, and suitability for high speed operation, and so on, and further the addition of multi-point positioning function allows this type of cylinder to have a far wider range of applications.

However, rodless cylinders have a rather large friction coefficient at their sliding portions owing to their structure, and consequently, when used for multipoint positioning, it causes problems such as the deterioration of positioning precision, increase of positioning time, and the like.

In this study, to overcome the above problems, and in order to realize a high speed pneumatic servo robot making the most of the features of the above 
rodless cylinder, and also to shorten the positioning time during deceleration, the improved pulse driving method is proposed in which the positioning is controlled by pulse driving method with pressure as switching condition from the viewpoint of practical application. This positioning method is embodied in experimental production of a cartesian coordinates 2-axis pneumatic servo robot, and its useful advantages are experimentally examined.

\section{CONFIGURATION OF PNEUMATIC SERVO ROBOT}

The experimentally produced robot is a cartesian coordinates 2-axis robot, whose specifications are shown in Table 1. Figure 1 shows the appearance of the experimental robot, and rodless cylinders are employed in its $\mathrm{X}$ - and $\mathrm{Y}$-axis. Figure 2 shows the configuration of the entire system, and the control portion comprises a host computer to communicate with robot or external devices and a servo computer to control positioning, enabling control of up to 4degrees-of-freedom. In the machine mainframe, the pressures of both the cylinder chambers are detected by the pressure sensors at both the ends of cylinder, and the displacement of the moving portion turns the pulley via the timing belt, and is detected by the rotary encoder.

Table 1 Specification of experimental pneumatic servo robot

\begin{tabular}{|l|c|}
\hline Maximum Velocity & $1.0 \mathrm{~m} / \mathrm{s}$ \\
\hline Potioning Accuracy & $\pm 0.1 \mathrm{~mm}$ \\
\hline Loadable Weight & $5.0 \mathrm{Kg}$ \\
\hline Stroke (X,Y-axis) & $\mathrm{X}: 500, \mathrm{Y}: 300 \mathrm{~mm}$ \\
\hline Cylinder (X,Y-axis) & $\mathrm{X}: \phi 32, \mathrm{Y}: \phi 25 \mathrm{~mm}$ \\
\hline
\end{tabular}

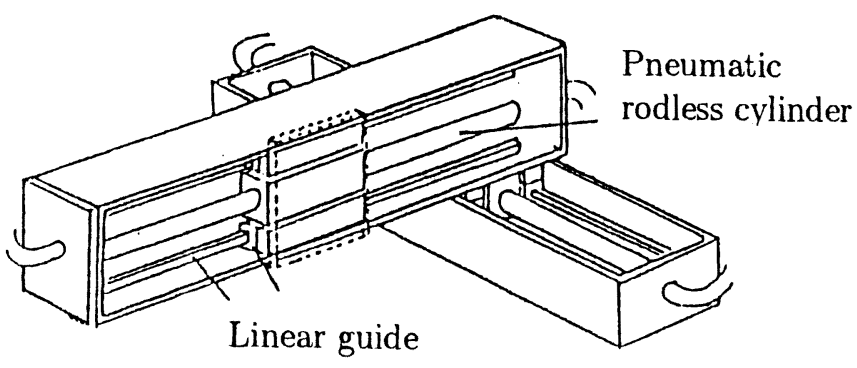

Figure 1. Configuration of experimental pneumatic servo robot

\section{POSITION CONTROL METHOD}

The proposed positioning control method, as shown in Figure 3, carries out positioning control by high speed driving from the start of action to the point $r 1$ just before the target point $r$, and by slowdown action by pulse driving near the target point.

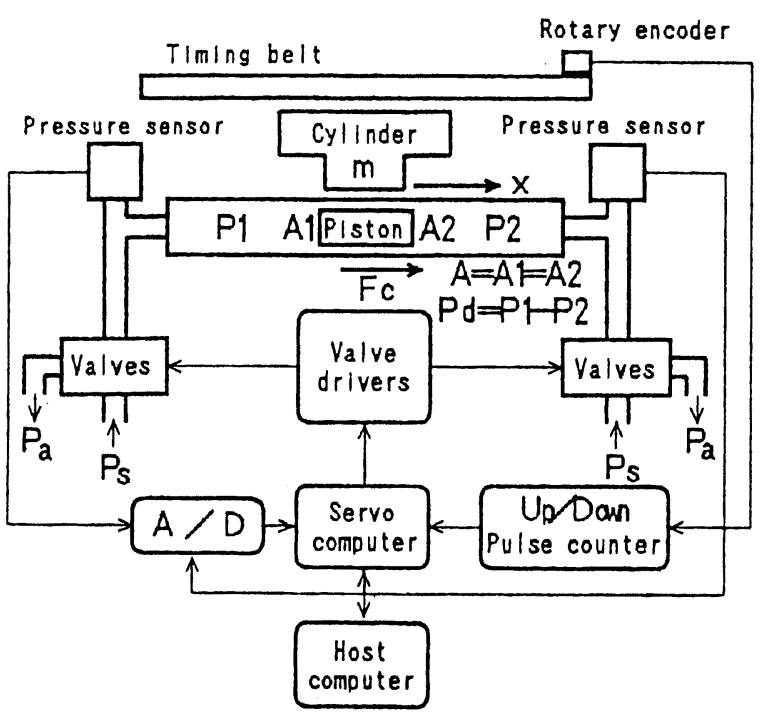

Figure 2. Experimental robot system in case of $\mathrm{X}$-axis

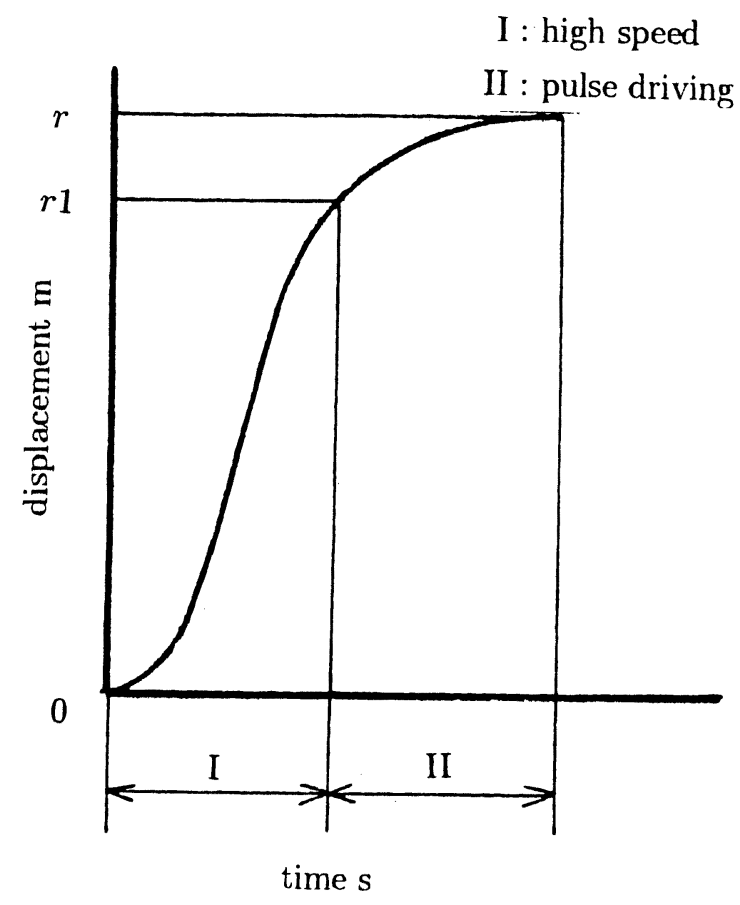

Figure 3. Position control scheme 
In the conventional pulse driving method, stable slow-down action may be realized and improved positioning precision may be expected[1]. However, if the action distance is long, positioning time increases owing to slow speed. Therefore, at the start of the action, one of solenoid valves is excited, and maximum driving force is given for high speed driving. Then, at a place near the target point, decelerated action is made by the pulse driving method in which the cylinder chamber pressure is used as the on-off switching condition of the solenoid valves. Thereby, low speed driving near zero is made just before the target point, and positioning precision is secured.

According to the present positioning control method, a control algorithm may be simply configured, and high level calculation capacity is not required for the computer, and low-priced on-off solenoid valves may be used. Therefore, the present control method is a low-cost and highly reliable control scheme from the viewpoint of practical applications.

Figure 4 shows a pneumatic driving circuit diagram. A 3-port solenoid valve with high speed response $(3.5 \mathrm{~ms}$ in terms of catalog value, effective cross section $9 \mathrm{~mm}^{2}$ ) is connected to each of the left and right cylinder chambers. Figure 5 shows the configuration of the control system. The sampling time appears to be $2 \mathrm{~ms}$, and the speed to be used as switching condition is obtained from displacement by use of difference calculation. Solenoid valves V1, V2 drive according to the control signal $u$ as follows:

$$
\begin{array}{lll}
u=U & : & \mathrm{V} 1=1, \mathrm{~V} 2=0 \\
u=-U & : & \mathrm{V} 1=0, \mathrm{~V} 2=1 \\
u=0 & : & \mathrm{V} 1=\mathrm{V} 2=1
\end{array}
$$

\section{(1: Air intake, $0:$ Air exhaust)}

\section{PULSE DRIVING METHOD}

In order to obtain far more stable control and far higher response than those with the conventional pulse driving method, the differential pressure $P d$ between both the cylinder chambers is used as the switching condition in the configuration of the control system.

The viscosity friction is small during the slowdown action, so it is negligible, and the action equation of driving system is expressed as shown below:

$$
m \ddot{X}=A P d-F c \cdot \operatorname{sgn}(\dot{X})
$$

where $m$ is mass of the moving portion, $X$ is displace ment of the moving portion, $A$ is effective sectional area of the piston, and $F_{C}$ is Coulomb's friction. And when the differential pressure $P c$ corresponding to Coulomb's friction $F c$ is expressed as $P c=F c / A$, $P e=P d-P c \cdot \operatorname{sgn}(\dot{X})$, then the above equation (1) is described as below:

$$
m \ddot{X}=A P e
$$

The switching condition of solenoid valve at pulse driving is expressed by the following equations:

In the case of $|X|>|e|$,

$u=-U$ :

$$
P d \geq P c \cdot \operatorname{sgn}(\dot{X})-K v(\dot{X}+C X)
$$

$u=U:$

$$
P d<P c \cdot \operatorname{sgn}(\dot{X})-K v(\dot{X}+C X)
$$

Wherein, the target point is $X=0, e$ is allowable positioning width, $K v$ is feed back gain, $C$ is slope of the switching line, and $K v>0, C>0$.

As a result, if the delay in pressure response of cylinder chamber is small, the following equation stands up (approximately):

$$
P d=P c \cdot s g n(\dot{X})-K v(\dot{X}+C X)
$$

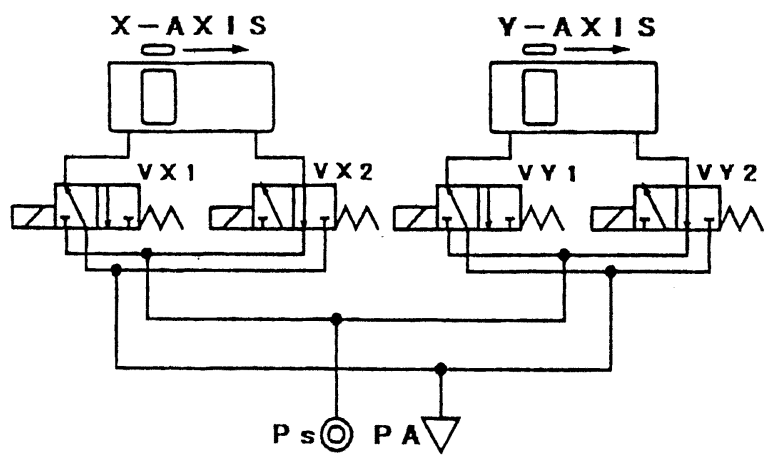

Figure 4. Pneumatic driving circuit

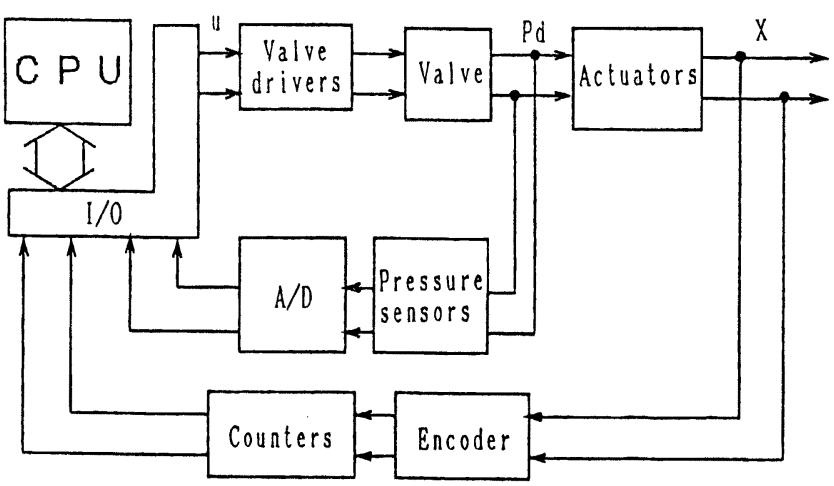

Figure 5. Experimental control system 
Namely,

$$
P e+K v(\dot{X}+C X)=0
$$

From the above equation (2), it leads to the following;

$$
\ddot{X}+\left(\frac{A K v}{m}\right) \dot{X}+\left(\frac{A K v C}{m}\right) X=0
$$

Consequently, the natural freqency of the system $\omega_{n}$ and damping coefficient of the system $\zeta$ are expressed by the following equations:

$$
\omega_{n}=\sqrt{\frac{A K v C}{m}}, \zeta=\frac{1}{2} \sqrt{\frac{A K v}{m C}}
$$

Therefore, by pulse driving by use of the switching condition equations (3) and (4), the control system to meet the equation (8) can be realized (approximately), and response can be improved by using $K v$ and $C$ as parameters.

By the way, in the case of $|X| \leq|e|, u=0$ is commanded, and air is supplied to both the cylinder chambers so that supplied pressures become equal to stop the piston.

\section{RESULTS OF EXPERIMENT}

The behavior at high speed action under the present control method is shown in Figure 6. I represents the time of high speed driving, while II represents the time of positioning control. The maximum speed goes up to $1 \mathrm{~m} / \mathrm{s}$, and a slight vibration can be seen in area II, nevertheless, deceleration is smooth and there is no impact upon stopping.

Next, as for the behavior during the action, the phase plane trajectory comprising of displacement $X$ and speed $\dot{X}$ is shown in Figure 7 . If the straight line $\dot{X}+C X=0$, obtained by making the seconds of the right sides zero in switching equations (3) and (4), is regarded as a switching line, speed increases rapidly from the position of displacement $X=0.1 \mathrm{~m}$, and speed deceleration starts at the top of the switching line, that is, before reaching at the deceleration area, and speed is confined to this line and reaches the origin. In the high speed range, it always stands up that differential pressure $P d>P c$, that is, $P e>0$, and from equation (3), switching of the solenoid valve starts from the lower area of the switching line where $\dot{X} \geq-C X-P e$, and in the low speed range, differential pressure $P d=P c$, i.e., $P e=0$, it is confined to the trajectory on the switching line.

Generally, in the control system using such a switching line, chattering owing to switching delay may be a problem. In particular, pneumatic system, the action delay at actuator portion is conspicuous.
However, the present control method, where deceleration starts before reaching to the deceleration area of the switching line, has little switching delay, therefore it is effective to reduce the above action delay. In other words, the present control method, using differential pressure, that is, the driving force as the switching condition, has an effect equivalent to acceleration feedback, and is far more effective in controlling chattering than methods where switching is carried out using only displacement and speed.

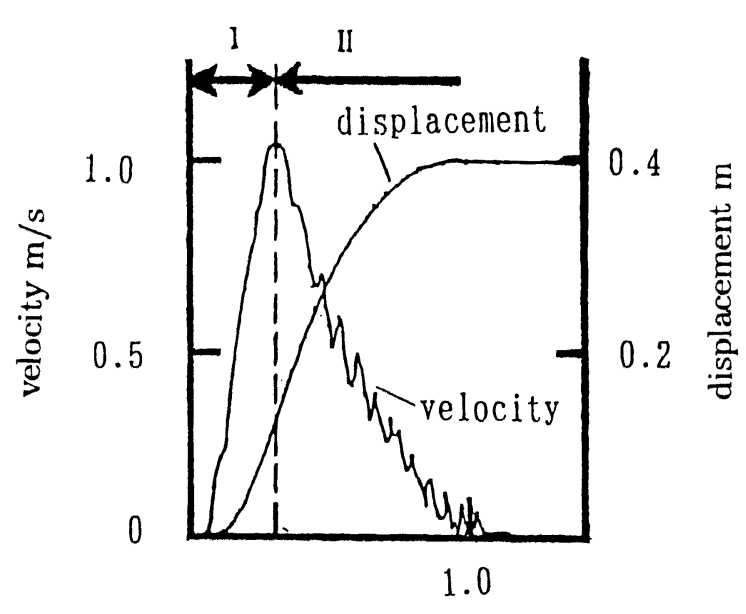

time $s$

Figure 6. Examples of step response $(P s=500 \mathrm{kPa})$

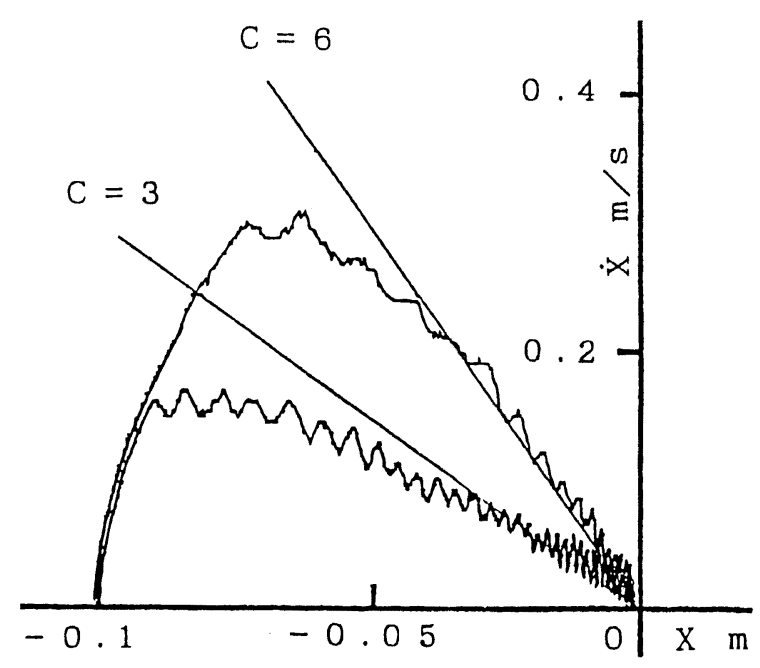

Figure 7. Phase-plane trajectories $(P s=500 \mathrm{kPa})$ 
Next, the behavior at the changes of parameters $C$ and $K v$, that is, at the changes of $\omega_{n}$ and $\zeta$, to determine the system characteristics is shown in Figure 8. In Figure 8 (a), the increase of $\omega_{n}$ accompanies the increase of quick response. And in Figure 8 (b), it is observed that making $\zeta$ small causes vibration. Therefore, it is confirmed that the characteristics of the control system $\omega_{n}$ and $\zeta$ can be varied by changing parameters $C$ and $K v$.

The relationship between total positioning time and positioning accuracy as the positioning performance of the present control method is shown in Figure 9 . The positioning action carries out 3 action modes, that is, $\mathrm{X}$-axis single action, $\mathrm{Y}$-axis single action, and $\mathrm{X}$ - and $\mathrm{Y}$-axis simultaneous action. The time difference from the start of action to the time when allowable positioning width $e$ is attained is the positioning time. In single action modes, the difference of positioning time is below 0.2 seconds with accuracy $0.1 \mathrm{~mm}$ to $0.5 \mathrm{~mm}$. This means that stable positioning is possible irrespective of positioning accuracy, and therefore, the presently proposed control method may be said to be effective for shortening the positioning time.

In $\mathrm{X}$ - and $\mathrm{Y}$-axis simultaneous action mode, as accuracy becomes small, the positioning time increases slightly more than in the case of $\mathrm{X}$-axis single action. This phenomenon is considered to be caused by the reduction of supplied pressure owing to simultaneous action or the influence by fluctuation, but details are to be examined further in the near future.

And it is considered that the difference between the positioning time of the $\mathrm{X}$-axis and that of $\mathrm{Y}$ axis is caused mainly by the difference of load mass, therefore, appropriate selection of pneumatic cylinder, solenoid valve, and so on may easily shorten the positioning time of the $\mathrm{X}$-axis.

\section{PNEUMATIC SERVO ROBOT}

On the basis of the position control method above, a positioning controller is developed and a large cartesian coordinates 2-axis robot is constructed using long-stroke rodless cylinders available on the market. Photo 1 shows the appearance of this large pneumatic servo robot, while Table 2 shows its specifications. Making the most of the features of rodless cylinders that although the weight of its moving portions such as piston and so on are rather light, a large cylinder internal diameter may be attained and the driving force is strong, the maximum velocity reaching at $1.5 \mathrm{~m} / \mathrm{sec}$. The positioning precision appears to be $\pm 0.5 \mathrm{~mm}$, which is rather rough compared with that of electric robots available commercially, but is sufficient for parts conveyance.

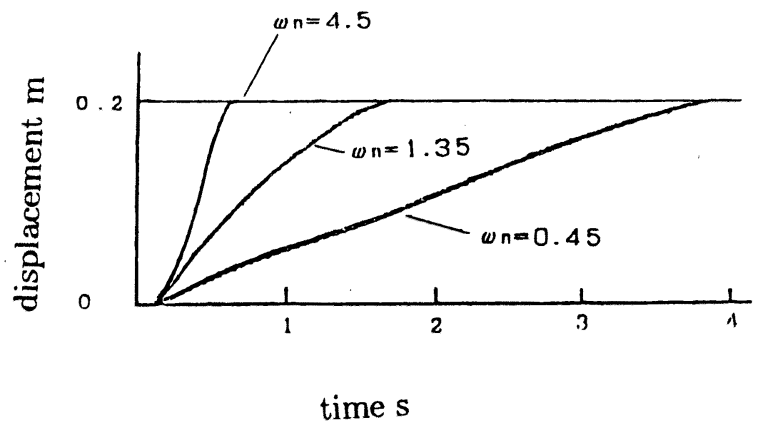

(a) Effect of $\omega_{n}$ with $\zeta=1.5$

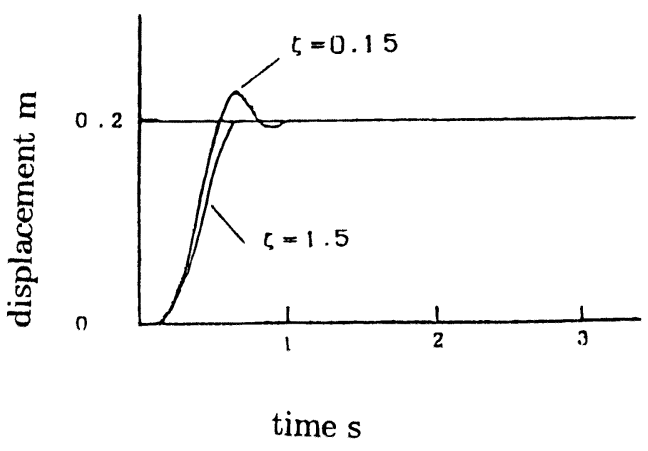

(b) Effect of $\zeta$ with $\omega_{n}=4.5 \mathrm{rad} / \mathrm{s}$

Figure 8. Effect of control parameters $C, K v$ on the step responses

$\left(P s=500 \mathrm{kPa}, A=0.000804 \mathrm{~m}^{2}, m=14.9 \mathrm{~kg}\right)$

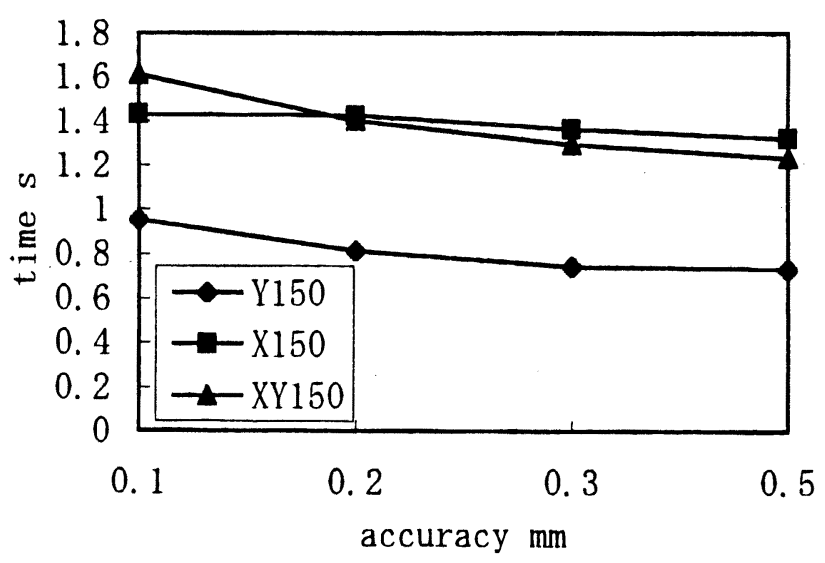

Figure 9. Relation between total positioning time and positioning accuracy with $C=1.5 \mathrm{~s}^{-1}$, $K v=25000 \mathrm{~Pa} \cdot \mathrm{s} / \mathrm{m}, P s=500 \mathrm{kPa}$ 
This large pneumatic servo robot was put in practical application in the parts packaging process in our factory, and became operative in 1993. And this robot has been manufactured in our own factory, and its construction cost is just about half that necessary for commercially available electric robots with a similar stroke and allowable load, and its action time is about $3 / 4$ that of commercially available robots, thus, offerring great benefits.

\section{CONCLUSIONS}

By using the pulse driving method in which the pressure is used as the switching condition, a positioning control method to maintain the positioning accuracy necessary for practical applications and to enable high speed action has been realized. And a large cartesian coordinates 2 -axis robot which moves at high speed and its cost is lower than electric robots available commercially was put in practical operations.

For the future, we plan to spread widely pneumatic servo robots and its positioning controller in product assembly lines by refining.

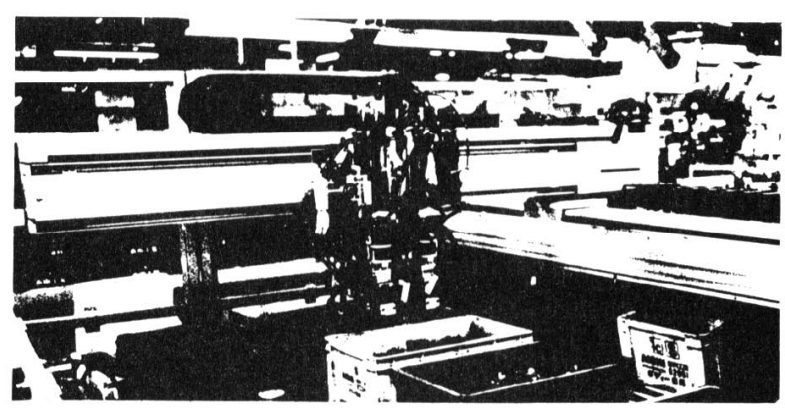

Photo 1. Pneumatic servo robot

Table 2 Specification of a practical large pneumatic servo robot

\begin{tabular}{|l|c|}
\hline $\begin{array}{l}\text { Number of axis, } \\
\text { Type }\end{array}$ & $\begin{array}{c}\text { cartesian coordinates } \\
\text { 2-axis type }\end{array}$ \\
\hline Maximum velocity & $\begin{array}{l}\text { X-axis } 1.5 \mathrm{~m} / \mathrm{sec} \\
\text { Y-axis } 1.5 \mathrm{~m} / \mathrm{sec}\end{array}$ \\
\hline Positioning accuracy & $\pm 0.5 \mathrm{~mm}$ \\
\hline $\begin{array}{l}\text { Maximum allowable } \\
\text { load }\end{array}$ & $15 \mathrm{kgf}$ \\
\hline Stroke & $\begin{array}{l}\mathrm{X} \text {-axis } 2000 \mathrm{~mm} \\
\text { Y-axis } 1100 \mathrm{~mm}\end{array}$ \\
\hline $\begin{array}{l}\text { Cylinder internal } \\
\text { diameter }\end{array}$ & $\begin{array}{r}\mathrm{X} \text {-axis equivalent } \\
\text { to } \phi 50 \mathrm{~mm} \\
\text { Y-axis equivalent } \\
\text { to } \phi 25 \mathrm{~mm}\end{array}$ \\
\hline
\end{tabular}

\section{REFERRENCE}

1. Toshiro Noritsugu: The Configuration of Electric and Pneumatic Servo Systems by Pulse Width Modulation Method, Theses of Measurement Automatic Control Academy Society, 20-8, 754/761, 1984. 